\title{
The \\ Th h he ema \\ Des échanges langagiers dans la classe pour construire des usages cognitifs du langage et réduire les inégalités scolaires
}

\author{
Élise Vinel, Université Paris 8, Laboratoire Circeft-Escol \\ Élisabeth Bautier, Université Paris 8, Laboratoire Circeft-Escol
}

\begin{abstract}
Au sein des classes, les démarches de construction des savoirs deviennent la norme et se substituent peu à peu aux pédagogies transmissives et frontales. Dès lors, les échanges élèves-élèves et enseignant.e-élèves sont très présents dans les classes, mais ils sont souvent ambigus et ne permettent pas à tous les élèves de réfléchir et de conceptualiser. Nous interrogeons ainsi la nature du discours produit au sein des classes, du point de vue des conditions et possibilités d'apprentissages dont ils sont porteurs, compte tenu des situations d'échanges mises en oeuvre par les enseignant.e.s. Nous nous appuyons dans nos analyses sur une recherche en cours portant sur l'enseignement de l'orthographe et de la grammaire, et le corpus recueilli à cette occasion.
\end{abstract}

\section{Le contexte contemporain des échanges langagiers en classe}

Au sein des classes, les démarches visant la construction des savoirs par les élèves sont de plus en plus fréquentes et se substituent peu à peu aux pédagogies transmissives et frontales. De nouvelles formes du travail scolaire en découlent ; les échanges élèves-élèves et enseignant-e-élèves sont très présents dans les classes du fait de l'organisation collective ou en groupe de l'élaboration des éléments de savoirs. Cette très grande présence de l'oral est motivée par l'influence des théories d'apprentissage et plus précisément de l'influence des théories socioconstructivistes (ou le plus souvent de leurs vulgates) comme celle de Vygoski (1934/1985) ou celle, post piagétienne, du conflit sociocognitif de Doise et Mugny (1981) ou encore des travaux comme ceux de Buchs et al. (2008) qui montrent l'influence des interactions sociales dans les progrès réalisés pour accomplir une tâche. Dans le même sens, des travaux plus directement didactiques (Orange, 2005, 2009) valorisent des approches qui sollicitent l'activité langagière et cognitive des élèves, dans des visées d'élaboration conceptuelle, en particulier la verbalisation que nécessitent la négociation et la confrontation.

Ces modalités d'apprentissage supposent le plus souvent des habitudes langagières chez les élèves à qui l'on demande alors de justifier, raisonner avec des savoirs disciplinaires, maîtriser les mots, notions et concepts et les spécificités des genres discursifs des différentes disciplines et les utiliser ; ces habitudes sont pourtant non partagées par toutes et tous et rarement enseignées (Bautier, 2001, 2005b). Ainsi que le souligne Lahire (1993), les démarches visant la construction des savoirs par les élèves, qui permettent aux élèves d'être en activité, de réfléchir sur le langage et la langue, sont plus adaptées aux élèves les plus proches de la culture scolaire, ceux qui peuvent « s'approprier les modes de discussion, les séquences discursives, c'est-à-dire comprendre ce qui est dit " (p. 153) et pourquoi c'est dit, ou encore identifier l'apprentissage visé. Bien que ces démarches soient « souvent présentées comme un moyen de "lutte contre l'échec scolaire" (qui serait principalement dû à la "rigidité", aux "formalismes", aux "contraintes" des méthodes "traditionnelles", "classiques")» (Lahire, 1993, p. 144), elles sont source de difficultés pour un certain nombre d'élèves qui ne parviennent pas à identifier les enjeux cognitifs des échanges, les « jeux de langage " sous-jacents, brouillés par les mises en situation proposées et entrainnant des malentendus (Bautier et Rochex, 1997). Ces différents usages du langage, auxquels on peut ajouter les échanges " conversationnels " de la classe, co-existent la plupart du temps lors des mêmes activités, sans que les enseignant·e.s, peu formé.e.s à ces distinctions, puissent identifier ce que tel.le ou tel.le élève engage le plus souvent avec le langage ou n'engage jamais (Bautier, 2005a, 2005b). Nos recherches (Bautier, 2001, 2005b) qui portent sur les relations entre les échanges langagiers et les apprentissages mettent ainsi en évidence une nécessité : l'importance de permettre à chaque élève d'être familier et familière des usages cognitifs du langage, ceux qui permettent de réfléchir sur l'objet d'enseignement et ce faisant d'apprendre dans le cadre scolaire.

Ces usages du langage à visée d'apprentissage, mobilisant des activités cognitives correspondent à ce que nous appelons ici la littératie scolaire. Comme les résultats des évaluations des compétences en littératie du programme PISA le mettent en évidence, ces usages du langage sont effectivement loin d'être partagés par 
l'ensemble des élèves de quinze ans, ce qui confirme la difficulté de leur apprentissage. L'évocation du PISA, bien qu'évaluant l'écrit des élèves, est pertinente dès lors que ce qui est travaillé ici, ce sont les usages littératiés du langage qui, dans les classes, alternent entre oral et écrit. Ce sont donc des habitudes langagières qui doivent être familières à l'oral et sans doute apprises à l'oral par les élèves afin que celles-ci puissent être (re)mobilisées à l'écrit. C'est d'ailleurs cette démarche que l'on retrouve dans les orientations des programmes scolaires de 2015, qui mettent en avant le travail d'explicitation par les élèves et le langage pour apprendre.

La littératie scolaire participe également de la construction de l'objet de savoir des disciplines scolaires ; comme l'indiquent les programmes officiels, les savoirs scolaires ne sont plus réductibles à leur seule définition ou énumération à restituer, mais sont le produit d'une construction, d'un questionnement élaborés dans le collectif de la classe et/ou des groupes d'élèves. La restitution des seules définitions ou règles, toujours nécessaires à connaître, ne semble plus suffire pour satisfaire les attendus scolaires quelles que soient les disciplines (pour l'exemple, la géographie qui vise actuellement à apprendre aux élèves « à penser le monde » et non plus à apprendre des définitions, voir Themines, 2016).

Afin d'approcher la façon dont le discours scolaire peut participer de la construction des inégalités, cet article interroge la façon dont les pratiques de classe et la nature des échanges que les enseignant.e.s y installent aident ou non les élèves à se familiariser avec ces usages du langage à visée d'apprentissage qui devraient donc faire l'objet d'un apprentissage particulier pour une grande partie des élèves. Il ne s'agit donc pas ici d'une recherche relevant d'une didactique disciplinaire, mais d'une étude portant sur ce qui, au moins potentiellement, peut aider les élèves à apprendre et mobilier des usages langagiers et cognitifs dont ils et elles sont initialement peu familiers et familières.

Nos exemples et nos analyses sont issus d'un corpus recueilli à l'occasion d'une recherche en cours sur l'enseignement de l'orthographe et de la grammaire, sans que ce soit cet enseignement en tant que tel de la grammaire et de l'orthographe qui nous intéresse ici. Il s'agit de la recherche "TAO-Twictée ${ }^{1}$ pour apprendre l'orthographe » dans le cadre du PIA ${ }^{2}$, comparant diverses pratiques d'enseignement-apprentissage de l'orthographe en classe. Nous avons sélectionné sept classes, quatre classes situées en réseau d'éducation prioritaire (REP) (1 CM1, 2 CM1-CM2, 1 CM2) et trois hors REP (3 CM2) qui ne pratiquaient pas la twictée (ce dispositif occasionnant des échanges sur les outils numériques utilisés, objet qui ne nous apparaissait pas pertinent pour notre étude sur les pratiques de classe et de discours scolaires potentiellement inégalitaires); d'où également le choix de comparer des classes en REP et hors REP. Pour chacune de ces classes, nous avons observé cinq séances d'enseignement/apprentissage de l'orthographe.

Nous avons choisi de nous intéresser à des situations d'enseignement de l'orthographe et de la grammaire car celui-ci est emblématique de ces nouveaux échanges dans la classe, à travers la mise en œuvre d'activités et de dispositifs dits " collaboratifs" (" dictées négociées ", "phrase dictée du jour ", "dictée zéro faute ", etc.), qui sont censés accorder une place privilégiée aux négociations entre élèves concernant le " choix » des bonnes formes, négociations supposées entraîner de leur part des explications, des justifications et des argumentations fondées sur les savoirs orthographiques et grammaticaux (Avezard-Roger et Thomas, 2017 ; Cogis et al., 2015; Fisher et Nadeau, 2014 ; Totereau et al., 2013). Même si tout se passe donc comme si l'intérêt et l'efficacité de ces activités et dispositifs ne semblent plus faire de doute et garantissent les apprentissages, enseigner l'orthographe selon des démarches inductives et faire procéder aux verbalisations par les élèves s'avèrent cependant des tâches difficiles à mettre en œuvre pour les enseignant.e.s (Péret et al., 2008 ; Fisher et Nadeau, 2014) comme pour les élèves.

Comme nous l'avons évoqué ces pratiques cognitives et langagières considérées comme un allant de soi, mettent pourtant une grande partie des élèves en difficulté de compréhension de la nature même de la tâche langagière et cognitive attendue (Bautier, 2016). Mais plus spécifiquement ici, même s'il importe d'engager les élèves dans ces usages cognitifs du langage, les difficultés de certain·ess, sans un étayage ciblé, risquent d'être renforcées par le fait de devoir parler sur la langue, de la prendre pour objet d'analyse et d'étude, d'observation, pour objet de travail scolaire. Cette objectivation et abstraction de la langue fait partie, selon nous, des composantes de la littératie scolaire, comme tout ce qui est transformation des objets du monde en objets de savoir

1 La twictée est un dispositif didactique innovant et numériquement outillé d'enseignement de l'orthographe. Dans ce dispositif, de courtes dictées sont l'occasion d'un travail de «négociation » au sein des groupes dans la classe, pour justifier les solutions aux problèmes orthographiques rencontrés, puis d'un échange via twitter avec d'autres classes pour proposer des corrections et les argumenter grâce à une catégorisation des erreurs élaborée par les élèves.

2 PIA : Programme d'investissements d'avenir du gouvernement français. 
ou encore ce que, à la suite de Bakhtine (1984), Jaubert et al. (2004) et Bautier (2005b) ont nommé "secondarisation ". Cette secondarisation est un usage spécifique du langage que certain·e-s élèves ne " fréquentent " qu'à l'école. Dans le même sens, et concernant le corpus étudié, penser la langue comme un système est un apprentissage nouveau à effectuer pour nombre d'élèves qui, confronté.e.s à la complexité de l'orthographe sont tenté.e.s de considérer ses réalisations comme aléatoires (Bautier, 1989).

Un autre exemple de difficultés rencontrées par une grande partie des élèves réside dans le passage, souvent supposé spontané par les enseignant·e.s, du mot entendu et compris comme une simple étiquette, à sa signification conceptuelle donc à l'ensemble des propriétés qu'il porte ; ce passage n'a pourtant rien d'automatique pour les élèves. On peut à ce propos faire référence aux travaux de Vygotski (1997/1934) : «L'apprentissage des mots et leur rattachement à des objets n'entraînent pas la formation d'un concept " (p. 194), il écrit plus loin que quand l'enfant utilise des concepts transmis par l'enseignant.e de manière magistrale, ce n'est qu'une "vaine assimilation des mots, un pur verbalisme, simulant et imitant chez l'enfant l'existence des concepts correspondants mais masquant en réalité le vide» (p. 277).

Pourtant en ce qui concerne l'étude de la langue au cycle 3, les programmes de 2015 sont clairs : la compréhension du fonctionnement de la langue est mise en avant plus que l'accumulation de savoirs orthographiques et grammaticaux. Les enseignant·e.s sont ainsi invité.e.s à faire raisonner, réfléchir les élèves sur les régularités de la langue, à leur faire prendre conscience que la langue fait système à travers l'analyse de corpus permettant les comparaisons, les manipulations, les transformations, les tris, les classements ; activités hautement littératiées. Il ne s'agit plus « seulement " de connaître les définitions et les règles d'accord mais d'avoir compris et construit les règles syntagmatiques qui gouvernent les relations entre les éléments de la langue. Comme le souligne Audion (2017), c'est bien « un changement de posture de l'élève qu'appellent les programmes : il faut prendre du recul par rapport à l'objet observé, étendre le champ des observables » (p. 42).

Si les travaux sur les pratiques de classe qui mettent en œuvre ces cadres théoriques et recherches en didactique de l'orthographe mettent en évidence des apprentissages effectifs (Sautot, 2016 ; Sautot et Geoffre, 2017), ces résultats supposent des conduites de classe adaptées et donc une formation des enseignant.e.s spécifique. Qu'en est-il alors quand des enseignant.e.s se saisissent "simplement " des préconisations contemporaines concernant l'importance des échanges langagiers des élèves et avec les élèves?

\section{Des échanges langagiers dans le contexte du travail grammatical et orthographique: deux types de pratiques de classe}

En référence aux caractéristiques de la littératie scolaire, aux programmes, aux observations de séances de classe que nous avons menées nous avons cherché dans une double démarche théorique et empirique à déterminer dans les classes :

- ce qui peut favoriser des usages cognitifs du langage et de la langue, et à l'inverse ce qui peut les entraver (questions ouvertes vs questions fermées, à réponse unique (Haas, 1999), dialogue à visée exploratoire vs jeu de questions-réponses, demande d'observation d'un corpus avec questionnement $v$ s exercices d'application d'une règle donnée à partir de données homogènes (Bulea Bronckart et al., 2017)),

- plus précisément dans les situations spécifiques étudiées (orthographe et grammaire) ce qui peut favoriser la construction de la langue comme un objet d'analyse et de questionnement et la sollicitation des élèves sur un registre d'élaboration d'une réflexion, d'un raisonnement appuyé sur des connaissances et des savoirs grammaticaux ou orthographiques,

- la nature des savoirs en jeu : ponctuels vs systémiques et permettant de " comprendre que la langue est un système où tous les éléments sont en relation "(Nonnon, 2010, p. 11),

- la nature et l'usage d'un métalangage en ce qu'il induit une signification de simple nomination (étiquette) ou conceptuelle,

- la nature de l'intervention de l'enseignant.e : simple régulatrice de l'activité ou instructrice (Bernstein, 2007),

- la nature de l'étayage langagier (Bruner, 1983) : l'étayage trop serré peut conduire à un " sur-ajustement " (faible exigence cognitive et sollicitation d'un savoir déjà là), l'étayage trop lâche à un " sous-ajustement " qui peut entraver le travail d'élaboration nécessaire (Bautier et Goigoux, 2004) et autoriser les malentendus sur les visées de la tâche (Bautier et Rochex, 2007),

- enfin, l'arbitrage donné sur les réponses des élèves : validation ou non de la réponse et justification dans l'un et l'autre cas. 
$\mathrm{Si}$, a priori, les conduites de classe peuvent apparaître très comparables car accordant toutes, ou presque, une place très importante aux échanges langagiers, l'analyse du discours scolaire des différentes classes étudiées et des échanges qui y ont lieu permet de mettre en évidence des pratiques d'enseignement qualitativement différentes du point de vue du travail cognitif sollicité chez les élèves et d'identifier différentes conduites du dialogue scolaire, évidemment en relation avec le type d'activités proposées aux élèves.

Ces différences de conduites discursives ne se définissent pas par la seule présence ou l'absence de tel ou tel élément ou par une seule de ses occurrences, mais par le cumul d'éléments qui, par leur récurrence et les logiques de travail cognitif et langagier similaires qui les sous-tendent, orientent ce travail des élèves vers un régime majeur ou mineur (Bautier et Rayou, 2013), en d'autres termes, plus près des attendus de la complexité de la littératie scolaire ou plus éloigné et élémentaire. Nous présentons ci-après deux conduites du dialogue scolaire emblématiques des différences qui apparaissent à l'analyse comme exemplaires des pratiques des enseignant.e.s concernée.es quels que soient l'objet et la visée des séances. La première conduite familiarise peu les élèves à la littératie scolaire, au contraire de la seconde.

Type 1 : Un type de dialogue engageant peu Le travail cognitif et langagier des élèves

L'échange ${ }^{3}$ ci-dessous, qui a eu lieu dans une classe de CM2, permet d'illustrer un type de pratiques que l'on peut rencontrer dans de nombreuses classes. Il s'agit de choisir entre deux terminaisons possibles (-é ou -er) pour l'écriture de verbes en -er.

ENS : donc on va continuer à travailler sur l'écriture des verbes en -er à savoir si on les écrit-e accent aigu ou -er + alors est-ce que vous pouvez me rappeler rapidement la règle?

ELE1 : quand c'est-e accent aigu et ben on dit vendu quand c'est -er on dit vendre

ENS : alors effectivement mais c'est pas tout à fait comme cela qu'il faut l'expliquer

ELE2 : c'est vendre et prendre

ENS : qui est-ce qui veut bien essayer d'expliquer autrement?

ELE2:-er c'est pour les verbes à l'infinitif

ENS : oui

ELE2 : et -e accent c'est pour les verbes au participe passé

ENS : particulièrement les?

Plusieurs ENF : les participes passés

ENS : les participes passés effectivement -- pour bien distinguer la lettre finale des verbes en -er donc on remplace par un autre verbe + nous on a choisi "vendre " ça peut être "prendre " aussi -- si on peut remplacer par le verbe à l'infinitif "vendre " le verbe en -er s'écrira -er puisquill sera à l'infinitif et sìl s'agit du participe passé il sécrira on pourra dire "vendu " et donc il sécrira -e accent aigu -- c'est bon ? on va refaire un exercice sur le cabier du jour rapide + pour vérifier + pour vérifier si vous avez compris puis après on rajoutera une petite difficulté d'accord? allez c'est parti

L'enseignante affiche au tableau numérique interactif (TNI) l'exercice à trous dont la consigne est : compléter par des verbes en -é ou -er.

ENS : donc là on fait un exercice d'application pour vérifier que tout le monde a bien compris + tout le monde a réutilisé la règle

ELE3 : la règle?

ENS : la règle + et une fois qu'on a corrigé et que tout le monde est au clair avec ça on peut complexifier un petit peu

La séance débute par l'annonce de l'objet de la séance à travailler "savoir si on les (les verbes) écrit -e accent aigu ou -er ». L'enseignante sollicite alors les élèves pour un rappel de la règle permettant d'établir l'écriture des verbes en -er, règle qui s'énonce sous forme de procédure automatique "quand c'est-e accent aigu et ben on dit vendu quand c'est -er on dit vendre ». La réponse donnée par un premier élève étant minimaliste, elle demande à d'autres d'expliquer autrement, dans un étayage sans doute trop lâche pour obtenir la réponse escomptée.

3 Le code de transcription utilisé est inspiré du Groupe d'Aixois de Recherche en Syntaxe (GARS). ENS désigne l'enseignante, $E L E$ désigne un.e élève ; les pauses brèves sont marquées par le signe +, les pauses plus longues par --. 
Des échanges ont alors lieu entre des élèves sur lesquels l'enseignante s'appuie pour énoncer à son tour la règle oralement à l'ensemble des élèves en vue d'une stabilisation "pour bien distinguer la lettre finale des verbes en -er donc on remplace par un autre verbe si on peut remplacer par le verbe à l'infinitif "vendre "le verbe en -er sécrira -er puisqu'il sera à l'infinitif et s'il s'agit du participe passé il s'écrira on pourra dire "vendu" et donc il s'écrira-e accent aigu ». Si le rappel d'une règle semble nécessaire pour débuter une activité et créer des liens avec des activités précédentes, la règle énoncée par les élèves et l'enseignante vise ici essentiellement l'application d'une procédure permettant l'écriture d'une bonne forme, donc l'orthographe, sans prise en compte du fonctionnement de la langue, à savoir la recherche du sujet qui accompagne le verbe, ou la présence d'un auxiliaire ou la conjugaison des différents types de verbe en fonction de leur terminaison infinitive. Il n'y a pas de problématisation engageant une réflexion, aucun questionnement sur ces différences de terminaison, sauf la question de savoir si c'est -er ou -e accent aigu.

Même si cette procédure est souvent efficace en situation, elle peut, comme nous avons pu le remarquer lors des entretiens métagraphiques qui ont accompagné nos observations, empêcher certain·e·s élèves de se demander, dans d'autres activités (production d'écrit ou dictée), si le verbe était conjugué ou pas et donc privilégier une terminaison en -é ou -er à celle d'une terminaison d'un verbe à l'imparfait ou à la seconde personne du pluriel au présent. Les métatermes "verbe ", "infinitif ", " participe passé " ne sont employés qu'en tant qu'étiquette (-er c'est pour les verbes à l'infinitif et -e accent c'est pour les verbes au participe passé).

L'enseignante souhaite ensuite "vérifier que tout le monde a bien compris " et donc que l'ensemble des élèves s'appuient sur cette " règle » énoncée oralement pour trouver les bonnes orthographes de verbe (mais sans jamais employer le terme "terminaison»). Manifestement, un élève n'a pas bien compris de quelle règle il s'agissait, et lorsqu'il s'aventure à poser une question montrant son incertitude "la règle? ", l'enseignante ne fait que la répéter par l'affirmative "la règle ", sans expliciter davantage. Les élèves s'exercent alors oralement à partir d'un exercice "à trous " projeté sur le TNI. Un ensemble de phrases sur le même modèle est affiché au tableau, l'enseignante demande systématiquement de remplacer le mot inachevé par "vendre » ou « vendu » et de donner les lettres finales. Les élèves interrogé.e.s lisent alors à voix haute chacune des phrases en ayant pris le soin d'énoncer en son entier le mot à compléter, donnent la terminaison puis reprennent la phrase en remplaçant le verbe en -er comme préconisé par « vendre " ou "vendu ».

Regarde ces armures elles ont traversé (vendu) les siècles.

N'oublie pas de bien fermer (vendre) la porte en partant.

Le skieur lancé (vendu) à grande vitesse semble prêt à s'envoler (vendre).

L'élève est allé (vendu) chercher (vendre) son professeur pour se faire aider (vendre).

Cette substitution paraît efficace dans cette situation et les élèves semblent peu gêné.e.s par le manque de sens des phrases ainsi obtenues. Ceci peut s'expliquer notamment par le fait que cette demande de substitution est faite par l'enseignante dans l'exercice, qu'ils et elles n'ont pas à repérer le verbe d'eux et elles-mêmes, mais qu'en sera-t-il lorsque les élèves se retrouveront seul·e·s, notamment ceux et celles les plus en difficulté, pour mettre en place cette procédure de remplacement ? Cette procédure, pour être efficiente dans d'autres situations parce que sous tendue par un apprentissage, devrait sans doute être assortie d'un raisonnement qui ne s'arrête pas au seul remplacement par un verbe en -dre, d'autres verbes en -ir ou en -oir peuvent être choisis pour plus de cohérence, mais également être utilisée dans d'autres situations où le verbe n'est pas déjà identifié, mais où il doit être repéré par l'élève, et donc où le choix entre -er ou -é doit être réfléchi par l'élève. L'utilisation mécanique de ce remplacement par un verbe en -dre conduit les élèves à utiliser le verbe "vendre » dans toutes les situations d'hésitation - même lorsqu'elle n'est pas pertinente - parce que sa présentation en tant que tactique automatisée pour éviter les erreurs orthographiques ne permet pas aux élèves d'en comprendre la légitimité : les verbes en -dre n'ont pas l'ambiguïté phonétique conduisant à confondre le participe et l'infinitif. En outre, comme le notent Le Levier et al. (2018) les élèves en difficulté qui n’ont pas nécessairement de « sens grammatical aboutissent très vite à des erreurs dans la mesure où, même s'ils repèrent la bonne forme à l'oreille, le lien qu'ils établissent avec la forme en [e] correspondante est purement arbitraire» (p. 79). Elles soulignent de plus que "l'application d'une technique ne peut être durablement efficace si elle n'est pas reliée à une forme de compréhension grammaticale " (p. 79). Utiliser systématiquement le verbe "vendre " ne permet pas de choisir la terminaison lorsque le passé composé est construit avec l'auxiliaire être et le sujet est au féminin, il permet simplement de savoir que la finale est en [e]. Lahire le remarquait déjà en 1993 :

Les automatismes ou les imitations qui peuvent donner l'illusion de la « réussite », permettent à ces élèves de produire des résultats même lorsqu'ils sont dépourvus des principes de production scolaire de ces résultats. Cependant, au moindre changement, l'automatisme apparaît au grand jour et cesse de faire illusion, montrant 
ainsi le faible degré de maîtrise de la situation scolaire ou, plus précisément, le caractère extrêmement contextualisé des savoirs acquis, ce faisant loin d'être des savoirs remobilisables dans d'autres situations et durables. (p. 192)

Par ailleurs, dans cette activité collective, l'enseignante pose des questions qui sont souvent des questions fermées ; lorsqu'elles sont ouvertes, elles incitent davantage les élèves à énoncer des procédures plutôt qu'à mettre en œuvre un raisonnement sur la langue. C'est un jeu de questions-réponses initié pour l'essentiel par l'enseignante dans lequel elle laisse les réponses non attendues d'élèves en suspens (elle n'arbitre pas), ne leur expliquant pas pourquoi elles ne conviennent pas, les explications fausses ou incomplètes des élèves ne pouvant donc être déconstruites et risquent d'être proposées de nouveau.

Ce court exemple d'une situation d'échanges langagiers, dont les caractéristiques se retrouvent de façon fréquente dans d'autres classes dans des situations diverses, permet de mettre au jour des pratiques de classe récurrentes ainsi que les pratiques discursives qui les accompagnent : la présence de procédures qui visent l'automatisation de leur utilisation plus que de véritables activités réflexives sur la langue et la présence de savoirs ; des phrases présentées aux élèves, pour identifier un fait grammatical ou une difficulté, construites sur un même schéma syntaxique et qui ne permettent pas la comparaison et la compréhension d'un phénomène ; des suites d'exercices d'application sous forme de phrases à trous qu'il faut compléter et qui constituent l'entier d'une séance plus que le réinvestissement dans des activités complexes et des contextes différents ; l'emploi de métatermes, lorsqu'ils sont présents, en tant qu'étiquettes données sans explication et sans utilisation de métalangage ; un étayage ne permettant pas aux élèves d'aller au bout de leur raisonnement ou de mettre en évidence des procédures fausses, des incompréhensions.

Ces pratiques de classe font illusion car elles ressemblent à des classes discutantes qui échangent autour de la langue et se conforment ainsi en apparence aux préconisations didactiques et institutionnelles concernant « l'oral pour apprendre» dans la classe et les pratiques « d'interaction». Cependant à l'analyse, compte tenu de la récurrence de ce type de traitement de la langue et des conduites de dialogue qui sont autant d'éléments qui ne permettent pas à l'ensemble des élèves de se saisir de ces situations d'échange pour réfléchir, analyser, raisonner à partir de connaissances et de savoirs, il est à craindre qu'elles ne participent de la co-construction des inégalités au regard de la littératie scolaire.

Type 2 : Un dialogue d'apprentissage et de familiarisation avec La

Littératie scolaire

La seule présence d'échanges langagiers, voire même d'emploi de métatermes, ne permet pas, comme nous venons de le voir, d'assurer un travail cognitif et réflexif chez les élèves. Le second type de dialogue scolaire, que nous décrivons ci-après, met en œuvre une toute autre cohérence dans les registres de travail sollicités et mobilise la quasi totalité des critères de la littératie scolaire. Il donne la possibilité aux élèves de réfléchir et de comprendre ce qu'est le fonctionnement de la langue, de mettre en ouvre des activités d'analyse de la langue dans sa complexité et sa généricité, éloignées d'exemples très particuliers ou de procédures élémentaires au profit d'un raisonnement sur et à propos de la langue. Compte tenu de l'objectif de nos analyses, nous développons plus longuement la description de ce type de dialogue potentiellement plus favorable aux apprentissages cognitivolangagiers des élèves tout en le comparant au type précédent.

Les éléments caractéristiques de ce dialogue et qui le différencient du précédent concernent :

- la conduite du dialogue : le rôle de l'étayage enseignant dans la construction des savoirs,

- la nature et la fonction des savoirs dans le discours produit,

- la langue des dialogues : des métatermes à la métalangue et au métalangage.

Mais l'analyse des différentes séances correspondant à ce type de dialogue a conduit à identifier deux autres éléments qui accroissent la cohérence du travail sollicité chez les élèves :

- la gestion du temps de la séance, de sa visée, de sa progression,

- le corpus de mots ou de phrases proposé à l'observation des élèves.

Nous nous appuierons sur l'exemple suivant pour illustrer une partie de notre propos, mais les phénomènes qui s'inscrivent dans la durée entière de la séance (une règle qui se construit progressivement, le point sur les savoirs qui sont ainsi construits peu à peu) ne peuvent apparaître dans le cadre de cet article.

Après une séance de production d'écrit, l'enseignante, qui a sélectionné quelques phrases comportant des fautes d'orthographe, souhaite que les élèves les repèrent et les corrigent. Les phrases sont écrites au tableau, la consigne est également écrite au tableau après avoir été énoncée par l'enseignante et reformulée par un élève. 
Les élèves recherchent les erreurs d'abord individuellement sans aide de l'enseignante et réécrivent les phrases "sans fautes " sur leur cahier. Ils et elles doivent justifier leurs corrections. Puis, l'enseignante, pour orienter le travail des élèves, souligne les fautes au tableau pour qu'en groupe, ils et elles puissent échanger sur une base commune. Elle insiste sur la nécessité de justifier leurs choix, et surtout sur l'importance de justifier les affirmations "pourquoi, pourquoi, pourquoi " dit-elle. Les fautes sont ensuite discutées et corrigées en collectif.

ENS : on réfléchit ensemble + vous levez le doigt + "Ils sont devenue des lutin historien " + alors?

ENF1 : "devenue " c'est $-s$

ENS : pourquoi?

ENF1 : parce que

ENS : vous êtes d'accord?

Plusieurs ENF : ouilnon

ENS : levez le doigt si vous n'êtes pas d'accord + il s'exprime après ce sera ton tour vas-y

ENF2 : moi je dis que c'est sans le -e parce que déjà c'est pas "elles sont devenus " et avec un -s parce que l'auxiliaire ou le verbe c'est "sont"

ENS : l'auxiliaire + c'est lequel auxiliaire? l'auxiliaire?

Plusieurs ENF et ENS : être

ENS : quel est le temps du verbe déjà / "sont devenus " quel est le temps?

Plusieurs ENF : participe passé

ENS : le passé composé + dans le passé composé on accorde avec l'auxiliaire être + on accorde avec quoi ? le participe passé on l'accorde avec?

Plusieurs ENF: un -s

ENS : avec le sujet + le sujet c'est "ils» + quel est le genre et le nombre?

ENF3 : le genre c'est masculin le nombre c'est pluriel

ENS : pluriel / donc pas de -e " devenus " -us + c'est bon?

ENS : "ils sont devenue des lutin historien" + alors qu'est-ce qui manque?

ENF4 : dans "lutin" il faut mettre un - s

ENS : un -s pourquoi?

ENF5 : parce que c'est " des"

ENS : et alors? et alors? j'accorde quoi avec quoi ? quelle est la nature du mot "lutin"?

Plusieurs enfants répondent mais c'est inaudible.

ENS : un "lutin" + la nature?

Plusieurs ENF : un nom

ENS : un nom commun + on accorde le nom commun avec son?

Plusieurs ENF : déterminant

ENS : déterminant + donc si le déterminant est au pluriel le nom commun est au?

Plusieurs ENF : pluriel

ENS : historiens?

Plusieurs ENF : -s

ENS : pourquoi?

ENF : parce qu'il s'accorde / il s'accorde avec le nom

Plusieurs ENF : c'est pluriel

ENS : d'accord c'est l'adjectif qui s'accorde avec le nom qui s'accorde avec le déterminant

La conduite du dialogue: Le rôle de L'étayage enseignant dans

La construction des savoirs

Un premier élément différencie les séances observées : la conduite du dialogue et l'étayage enseignant. En effet, si l'on suit Sève et Ambroise (2009), la conduite du dialogue permet à l'enseignant.e d'observer comment les élèves se représentent le système orthographique. Pour cela, l'enseignant·e, non seulement s'adresse au collectif de la classe, mais également prend en considération les verbalisations individuelles des élèves, en poussant chacun.e à aller jusqu'au bout de son raisonnement. Ce dernier point, essentiel aux apprentissages, se retrouve dans ce second exemple alors qu'il s'avère absent du premier. Pour faire avancer les élèves dans la construction des savoirs, l'enseignante dans ce second exemple pose des questions ouvertes qui suscitent une observation de la langue et la réflexivité, demande sans cesse aux élèves de justifier leur réponse, d'aller jusqu'au bout de leur raisonnement par des "pourquoi ? ", " et alors ? ", mais aussi des questions qui demandent des verbalisations 
plus précises qui s'appuient sur des savoirs et des mots du savoir. Ici, l'étayage langagier de l'enseignante, mené à partir d'interventions explicites, conduit l'ensemble des élèves à un questionnement, puis à la formulation des raisonnements orthographiques avec emploi de "si » et de " donc " dans le discours enseignant afin de trouver les régularités de la langue et la règle générale. Ces exemples de modalités de raisonnement produits par l'enseignante sont pertinents car, ainsi que le notent Combaz et Elalouf (2015), "il est délicat de co-construire un raisonnement complet en justifiant sa finalité et chacune de ses étapes et en utilisant un métalangage partagé » (p. 108). Cet étayage serré guide le travail de réflexion des élèves. Il est à distinguer d'un étayage très étroit qui ne porte attention qu'à des points très précis limitant le travail des élèves à de simples réponses à des questions fermées et qui va de pair avec une absence de progression dans les apprentissages entre le premier et le dernier moment de la séance.

Pour mettre en place ce type de dialogue et d'étayage, comme le rappellent Lepoire et Sautot (2009), il importe que les enseignant.e.s perçoivent que l'activité cognitive et langagière de leurs élèves est essentielle pour leurs apprentissages et c'est sans doute ce qui fait la différence entre les deux types de dialogue.

Cette conduite du dialogue qui provoque chez les élèves des sollicitations différentes dans la nature du travail cognitif et langagier produit (par exemple, se poser des questions, analyser un corpus, faire des hypothèses, proposer des solutions, verbaliser), contrairement à la conduite précédente, va bien au-delà d'un jeu de questions-réponses.

La nature et la fonction des savoirs dans le discours produit

Un deuxième élément qui différencie ces deux dialogues concerne la nature et la fonction des savoirs dans le discours produit. Les savoirs présents en classe sont généralement de deux types : contextualisés et/ou élémentaires (définitions, règles, procédures) vs génériques et problématisés. Dans ces exemples de l'enseignement de l'orthographe ou de la grammaire, nous avons distingué des savoirs ponctuels (élémentaires), qui correspondent à des savoirs méthodologiques, procéduraux, qui sont très contextualisés, liés à des exercices ou situations spécifiques et des savoirs systémiques, qui sont introduits à la suite d'un questionnement, d'une problématisation qui conduisent à la présentation des concepts, ou au moins des notions (génériques).

Dans le premier exemple, savoir choisir entre -é et -er dans un type d'exercice particulier par l'utilisation d'une procédure ne garantit pas, nous l'avons vu, de savoir choisir en production d'écrit ou en dictée parmi l'ensemble des possibilités d'écriture du son [e] la bonne terminaison d'un verbe du premier groupe. Seul.e.s les élèves qui ont compris le but et la logique du remplacement pourront y parvenir ou participer aux échanges collectifs et répondre dans d'autres situations aux questions posées par l'enseignante. Les seconds types de savoirs, même quand ils correspondent pour partie à des savoirs " déjà là ", sont pour l'enseignant.e non seulement un moyen de vérifier leur présence et de les stabiliser, mais aussi de montrer aux élèves la progression de l'apprentissage. En effet, ces savoirs sont alors nécessaires pour construire les raisonnements ou observations qui visent à faire progresser les élèves dans l'élaboration d'une nouvelle connaissance.

Si les élèves ne sont jamais confronté.e.s au second type de dialogue, nous formons l'hypothèse qu'ils et elles ne peuvent acquérir l'habitude d'élaborer par eux et elles-mêmes une réflexion à partir d'observations et de questionnements, quelle que soit la discipline scolaire concernée; ce qui peut expliquer leur difficulté récurrente à satisfaire les exigences de littératie scolaire, à 15 ans encore (voir l'évaluation du PISA).

La nature des questions de l'enseignante, mais également les activités ou exercices proposés, vont ainsi de pair avec des mobilisations différentes de savoir chez les élèves. Les questions à réponse unique, comme les exercices d'application sollicitent principalement des savoirs (y compris procéduraux) déjà présents, acquis antérieurement (y compris les années précédentes). Les questions ouvertes sollicitant la réflexion des élèves visent non seulement des savoirs déjà là, mais aussi la construction de savoirs nouveaux pour les élèves.

La construction de règles générales, qui est le but de cette seconde conduite du dialogue scolaire, permet plus facilement aux élèves de distinguer les deux types de savoirs et la façon dont il est possible de construire de la généricité à partir d'exemples. Dans le type de dialogue précédent, les " savoirs » issus des exercices d'application sont restés ancrés dans cette utilisation ponctuelle ; nous mettons ici des guillemets au mot " savoir " car il est rare en ce cas qu'après des exercices d'application d'une procédure, une règle générique soit formulée.

Dans la dynamique des échanges du second type de dialogue, l'enseignante (ou les élèves) rappelle(nt) sous la forme de règles des savoirs censés être déjà acquis afin que les élèves s'inscrivent dans des raisonnements systématiques ; l'enseignante peut également formaliser et stabiliser des savoirs nouveaux soit au cours de l'activité afin que les élèves puissent continuer d'avancer dans la résolution du problème qui fait l'objet de la séance, soit en fin d'activité pour stabiliser l'ensemble des savoirs. 
La Langue des dialogues: des métatermes à la métalangue et au métalangage Un troisième élément concerne l'usage d'un métalangue et d'une métalangage plus que de métatermes. Si la plupart des enseignant.e.s mais aussi des élèves utilisent des métatermes (du type nom, verbe, complément, etc.), leurs usages sont cependant à différencier. Là où dans le premier type de dialogue, il ne s'agit que de nomination, d'étiquettes pour nommer les catégories de la langue et les fonctions, dans les dialogues d'apprentissage (second type présenté), cette nomination est complétée par l'explicitation des relations entre ces catégories et de leurs propriétés en utilisant pour ce faire des mots de l'analyse de la langue (accord, pluriel - et non " il y a un $s$ ", sujet, complément, etc.), et ce de façon récurrente voire systématique. Dans ce second cas, l'accent est mis sur le caractère structural et non aléatoire de la langue et de l'orthographe. Les métatermes ne sont pas seulement des étiquettes mais prennent peu à peu valeur de concept et de propriété (Bulea Bronckart et al., 2017). À ces métatermes, s'ajoute la familiarisation avec un usage métalinguistique de la langue, usage important pour constituer la langue en objet d'analyse.

L'usage de métatermes peut être certes les prémices d'un usage plus métalinguistique de la langue, mais pour qu'il y ait véritablement continuum entre ces deux usages, il importe, comme nous l'avons rappelé précédemment, que l'enseignant.e aide les élèves à entrer dans ce dernier usage de la langue où les mots ne désignent pas «seulement " un référent sensible mais un ensemble de propriétés et présentent à ce titre une nécessité. Dans le cas contraire, comme nous pouvons le voir dans le premier type de dialogue, seul.e.s les élèves les plus familier-e.s de cet usage métalinguistique de la langue du fait de leur socialisation familiale pourront satisfaire les exigences de la littératie scolaire.

\section{La gestion du temps de la séance, de sa visée, de sa progression}

Un quatrième élément qui différencie fortement les deux pratiques de classe concerne la gestion du temps de la séance, son cadrage, sa visée, sa progression, éléments qui assurent la continuité du travail et de la réflexion des élèves et la cohérence de la séance. Dans cette séance, comme dans d'autres dans lesquelles les élèves raisonnent et où la langue devient objet de réflexion et d'analyse :

- la séance est cadrée car elle débute par une réflexion sur la démarche qui va être mise en œuvre (lien avec la séance précédente, objectif de la séance en cours et modalités du travail présentés) et sa justification explicite (erreurs produites en production d'écrit) et non, comme dans le premier exemple par la seule annonce de l'objet de la séance, savoir si on écrit -er ou -é à la fin des verbes, avec un simple rappel de la procédure à appliquer,

- la séance progresse vers un but : la résolution de problèmes initialement posés par l'observation et l'analyse d'un corpus de phrases ou de mots,

- les objets grammaticaux à travailler sont complexes et problématisés ; ils sont présentés explicitement comme la construction d'une régularité générale,

- la règle travaillée est à construire ; cette construction s'inscrit dans une dimension temporelle qui est celle de la séance elle-même et de l'activité toute entière. Par exemple dans une autre séance observée, pour comprendre comment se conjuguent les verbes à l'imparfait, il ne s'est pas agi d'apprendre seulement les terminaisons de l'imparfait, mais d'élaborer la règle de construction de l'imparfait à partir des infinitifs des verbes et, plus particulièrement, d'identifier la distinction entre la marque de l'imparfait et celle des personnes.

La séance se construit sur un temps long, par cheminement, elle se distingue d'une séance (comme celle de l'exemple précédent) dans laquelle la règle est donnée, est identifiée à une procédure automatisable et se poursuit par des exercices d'application. On peut identifier dans cette gestion du temps didactique, un élément de la pratique de classe qui renvoie à une conception du travail de l'élève et de son activité sous-tendant l'élaboration progressive des savoirs.

\section{Le corpus de mots ou de phrases proposé à l'observation et l'analyse des él èves}

Le cinquième élément de différence des pratiques concerne le corpus de mots ou de phrases proposé à l'observation et l'analyse des élèves

L’enseignante présente aux élèves un corpus de plusieurs phrases différentes - ou plusieurs mots selon l'objet de l'analyse - (et pas seulement deux phrases ou deux mots) pour leur permettre de réaliser des comparaisons, de se poser des questions, d'établir des liens entre les éléments de la phrase, d'établir des régularités ou des variations liées au contexte, puis des règles, en attirant l'attention sur des éléments du corpus afin d'orienter 
la réflexion de l'élève. Il s'agit alors de placer les élèves face à une situation-problème dont la solution exige un travail de recherche pour les élèves. Travailler sur un corpus d'erreurs d'élèves est plus favorable à la réflexion des élèves que la seule application d'une procédure automatisée comme dans l'exemple traité précédemment où l'enseignante propose quelques phrases dans lesquelles les élèves n'ont qu'à choisir entre deux terminaisons possibles pour compléter les terminaisons de verbes. Le choix de la réponse au hasard pouvant être un choix alors fréquent chez les élèves.

Dans le cas de la séance sur les terminaisons de l'imparfait, les élèves ont autant de verbes du premier groupe que du second et troisième groupe à observer, à analyser et à comparer dans le but de montrer les régularités des terminaisons de ce temps, les marques de temps et de personnes sont alors étudiées.

Le but de ces séances est de problématiser les apprentissages pour faire réfléchir les élèves en observant la langue, satisfaisant ce faisant les exigences de la littératie scolaire quant à la nécessité de compréhension du fonctionnement de la langue, de ses régularités et non pas d'appliquer des règles et des procédures proposées par l'enseignant.e.

\section{Conclusion}

Cette présentation des différences entre deux conduites du dialogue scolaire permet de mettre en évidence que malgré des éléments communs à toutes les classes d'aujourd'hui, les analyses de ces dialogues dans la perspective des usages littératiés de la langue et du langage font apparaître des différences importantes. Certes, nos analyses très contrastées peuvent paraître caricaturales et correspondre peu à la réalité de ce qui peut se produire dans la classe au quotidien. La première conduite de dialogue n'a rien d'exceptionnel et correspond à des manières de faire récurrentes chez de nombreux enseignant·es, qui y voient des façons d'aider leurs élèves. La seconde est sans doute plus rare dans sa cohérence d'ensemble.

Cette contribution qui porte sur les questions de co-construction des inégalités au sein des classes vise à mettre au jour des éléments heuristiques concernant les logiques enseignantes à l'œuvre au quotidien qui cumulées construisent ces inégalités :

Les préconisations institutionnelles et didactiques concernant les échanges dans les classes, pour être fondées sur le plan théorique, ne sont pas aisées à mettre en œuvre, elles sont d'une grande exigence et supposent donc une formation solide dans le domaine des analyses et usages du langage. Les enseignant.e.s sont ainsi souvent tenté·e.s, même à leur insu, de " choisir » ou de ne retenir que tel ou tel aspect.

Nous l'avons vu, il ne s'agit pas seulement pour les enseignant.e.s de valoriser l'oral dans la classe, mais bien de mettre en œuvre une conception cognitivement exigeante de ce que doit être le travail de l'élève. Il est donc question ici d'une conception de l'enseignement lui-même, conception sans doute à construire en formation.

Il ne s'agit pas davantage d'avoir une conception minimaliste de l'oral à l'œuvre dans l'enchaînement d'un jeu de question-réponse, qui favorise des échanges au cours desquels les élèves répondent, parfois par un mot, à des questions. Il importe au contraire de parvenir à la production de véritables interactions d'apprentissage, au sens d'une activité simultanément langagière et cognitive supposant la résolution par le langage de conflits socio-cognitifs permettant le développement et la stabilisation d'un apprentissage (Vygotski, 1985/1934), cette résolution s'appuyant en classe sur des savoirs disciplinaires. Mais alors il s'agit sans doute aussi de former les enseignant.e.s à l'analyse des dialogues scolaires afin de les aider à identifier des situations d'oral pertinentes.

Nous espérons avoir montré que ce n'est pas l'existence de tel ou tel élément qui suffit à faire réfléchir et raisonner les élèves, mais que c'est la cohérence d'une démarche exigeante grâce au cumul de l'ensemble des caractéristiques des conduites dialogiques décrites dans le second type de dialogue, pratiquées de façon récurrente et systématique, qui peut conduire au développement chez les élèves de l'habitude de l'analyse des données et du raisonnement, en particulier chez ceux et celles qui sont les moins familiers et familières en dehors de l'école de ces usages simultanément cognitifs, linguistiques et langagiers.

Certes, la fréquentation de dialogues du second type ne peut garantir pour tous les apprentissages cognitivolangagiers, mais nous faisons l'hypothèse qu'il s'agit là d'une conduite de dialogue nécessaire et que sans cette fréquentation régulière et dans la durée, ces apprentissages n’ont que peu la possibilité de se réaliser.

Nous pouvons conclure sur une note optimiste, en signalant que l'exemple du type deux est justement issu des enregistrements effectues en REP+. 


\section{Bibliographie}

Audion, L. (2017). Encourager la posture métalinguistique des élèves de cycle : Pourquoi ? comment ?. Le français aujourd'hui, 3, 39-52.

Avezard-Roger, C., et Thomas, I. (2017). L'accord du verbe aux cycles 2 et 3 : posture réflexive et médiation entre pairs. Le français aujourd'hui, 198, 91-112.

Bakhtine, M. (1984). Esthétique de la création verbale. Éditions Gallimard.

Bernstein, B. (2007). Pédagogie, contrôle symbolique et identité : théorie, recherche, critique. Presses Université Laval.

Bautier, É. (1989). Aspects socio-cognitifs du langage. Quelques hypothèses. Langage et société, 47, 55-84.

Bautier, É. (2001). Pratiques langagières et scolarisation. Revue française de pédagogie, 137, 117-161.

Bautier, É. (2005a). Mobilisation de soi, exigences langagières scolaires et processus de différenciation. Langage et société, 111, 51-72.

Bautier, É. (2005b). Formes et activités scolaires, secondarisation, reconfiguration, différenciation sociale. In N. Ramognino, P. Vergès, (dir.), Le Français hier et aujourd'hui. Politiques de la langue et apprentissages scolaires. Hommage à Viviane IsambertJamati (p. 49-68). Publications de l'Université de Provence.

Bautier, É. (2016). Et si l'oral pouvait permettre de réduire les inégalités ? Les dossiers de sciences de l'éducation, 36, 109-29.

Bautier, É., et Goigoux, R. (2004). Difficultés d'apprentissage, processus de secondarisation et pratiques enseignantes : une hypothèse relationnelle. Revue française de Pédagogie, 148, 89-100.

Bautier, É., et Rayou, P. (2013). La littératie scolaire, exigences et malentendus. Les registres de travail scolaire. Éducation et didactique, 2(7), 29-46.

Bautier, É., et Rochex, J.-Y. (1997). Ces malentendus qui font les différences. In J.-P. Terrail (dir.), La scolarisation de la France. Critique de l'état des lieu (p. 105-122). La Dispute.

Buchs, C., Darnon, C., Quiamzade, A., Mugny, G., et Butera, F. (2008). Conflits et apprentissage. Régulation des conflits sociocognitifs et apprentissage. Revue française de pédagogie, 163, 105-125.

Bulea Bronckart, E., Marmy Cusin, V., et Panchout-Dubois, M. (2017). Les exercices grammaticaux dans le cadre de l'enseignement rénové du français : usages, problèmes, perspectives. Repères, 56, 131-149.

Bruner, J. S. (1983). Le développement de l'enfant : savoir faire, savoir dire. Presses universitaires de France.

Cogis, D., Fisher, C., et Nadeau, M. (2015). Quand la dictée devient un dispositif d'apprentissage. Glottopol. Revue de sociolinguistique en ligne, 26, 69-91.

Combaz, C., et Elalouf, M.-L. (2015). Une phrase dictée, trois enseignant.e.s, trois formes d'étayage. Glottopol. Revue de sociolinguistique en ligne, 26, 92-110.

Doise, W., et Mugny, G. (1981). Le développement social de l'intelligence. FeniXX.

Fisher, C., et Nadeau, M. (2014). Usage du métalangage et des manipulations syntaxiques au cours de dictées innovantes dans des classes du primaire. Repères, 49, 169-191.

Haas, G. (1999). Les ateliers de négociation graphique : un cadre de développement des compétences métalinguistiques pour des élèves de cycle 3. Repères, 20, 127-142.

Jaubert M., Rebière R., et Bernié J.-P. (2004). Signification et développement : quelle "communauté ? "Raisons Éducatives, 85-104.

Lahire, B. (1993). Culture écrite et inégalités scolaires. Sociologie de l'«échec scolaire» à l'école primaire. Presses Universitaires de Lyon.

Lepoire-Duc, S., et Sautot, J.-P. (2009). La grammaire : un problème pour les élèves, un problème pour le maitre aussi. Repères, $39,125-142$.

Le Levier, H., Brissaud, C., et Totereau, C. (2017). L’accord du participe passé : une difficulté persistante en fin de scolarité obligatoire. In B. Kervyn, C. Brissaud, et M. Dreyfus (dir.), Repenser l'écriture et son évaluation au primaire et au secondaire (p. 63-86). Presses universitaires de Namur.

Nonnon, É. (2010). La notion de progression au cœur des tensions de l'activité d'enseignement. Repères, 41, 5-34.

Orange, C. (2005). Problématisation et conceptualisation en sciences et dans les apprentissages scientifiques. Les sciences de l'éducation-pour l'ère nouvelle, 38(3), 69-94.

Orange, C. (2009). La place des activités langagières en classe de sciences : une question de point de vue sur la science. In P. Schneeberger, et A. Vérin (dir.), Développer des pratiques d'oral et d'écrit en science (p. 17-27). INRP.

Péret, C., Sautot, et J.-P., Brissaud, C. (2008). Les professeurs entrant dans le métier et la norme orthographique. In C. Brissaud, J.-P. Jaffré, et J.-C. Pellat (dir.), Nouvelles recherches en orthographe (p. 203-214), Lambert Lucas.

Programmes d'enseignement du cycle des apprentissages fondamentaux (cycle 2), du cycle de consolidation (cycle 3) et du cycle des approfondissements (cycle 4). NO : MENE1526483A

arrêté du 9-11-2015 - J.O. du 24-11-2015 MENESR - DGESCO MAF 1. Extrait de : MENESR. «Bulletin officiel spécial n 11»

Sève, P., et Ambroise, C. (2009). Images, ciseaux, tirettes...Un exemple de bricolage didactique au CE1 autour des relations nom/ verbe. Repères, 39, 103-123.

Sautot, J.-P. (2016). Mesure du progrès en orthographe grammaticale. Compte rendu de recherche. Espe de Lyon, Inspection académique du Rhône, France. http://halshs.archives-ouvertes.fr/halshs-01348572

Sautot, J.-P., et Geoffre, T. (2017). De quoi la négociation graphique est-elle l'exercice ? Repères, 56, 73-90.

Themines, J.-F. (2016). La didactique de la géographie. Revue française de pédagogie, 197, 99-136.

Totereau, C., Brissaud, C., Reilhac, C., et Bosse, M.-L. (2013). L'orthographe grammaticale au collège : une approche sociodifférenciée. Approche Neuropsychologique de l'Apprentissage de l'Enfant, 123, 164-171.

Vygotski, L. S. (1934/1985). Langage et pensée. Éditions Sociales.

Mots-clés : Échange ; élève ; enseignant·e ; interaction ; métalangage ; verbalisation 


\section{Sprachbezogene Interaktionen im Klassenraum zur Förderung der kognitiven Nutzung von Sprache}

Zusammenfassung

Beim Unterrichten werden konstruktivistische Lehransätze zunehmend zur gängigen Praxis und ersetzen so allmählich transmissive und frontale Formen des Lehrens. Infolgedessen werden Schüler-Schüler- und LehrerSchüler-Interaktionen im Klassenraum präsenter. Diese sind jedoch häufig mehrdeutig bzw. unklar und erlauben es deshalb nicht allen Schüler/innen, angemessen zu reflektieren und konzeptuelles Wissen aufzubauen. Wir untersuchen im Folgenden die Art des in den Klassen produzierten Diskurses vor dem Hintergrund der Lernbedingungen und -möglichkeiten, die sich unter den von den Lehrenden implementierten interaktiven Lehr-Lernszenarien beobachten lassen. Unsere Analysen basieren auf aktuellen Untersuchungen zur Gestaltung der Vermittlung von Rechtschreib- und Grammatikunterricht.

Schlagworte: Austausch; Schüler; Lehrer; Interaktion; Metasprach ; Verbalisierung

\section{Scambi linguistici in classe per costruire usi cognitivi del linguaggio e ridurre le disuguaglianze educative}

Riassunto

All'interno delle classi, i processi di costruzione della conoscenza stanno diventando la norma e stanno gradualmente sostituendo le pedagogie trasmissive e frontali. Di conseguenza, gli scambi allievo-allievo e insegnanteallievo sono molto presenti nelle classi, ma spesso sono ambigui e non consentono a tutti gli alunni di riflettere e concettualizzare. Mettiamo quindi in discussione la natura del discorso prodotto all'interno delle classi, dal punto di vista delle condizioni di apprendimento e delle possibilità che esse apportano, date le situazioni di scambio messe in atto dagli insegnanti. Nelle nostre analisi attingiamo alle ricerche in corso sull'insegnamento dell'ortografia e della grammatica e al corpus raccolto in questa occasione.

Parole chiave: Scambio, studente ; insegnante ; interazione ; metalinguaggio ; verbalizzazione

\section{Language exchanges to build learning. Under what conditions ?}

\section{Summary}

Within classes, processes of building knowledge are becoming the norm and are gradually replacing transmissive and frontal pedagogies. Consequently, student-student and teacher-student exchanges are very present in classes, but they are often ambiguous and do not allow all students to think and conceptualize. We thus question the nature of the discourse produced within classes, from the point of view of the learning conditions and possibilities they bring, given the exchange situations implemented by teachers. Our analyses draw on an ongoing research on the teaching of spelling and grammar, and the data collected on this occasion.

Keywords: Exchange; student; teacher; interaction; metalanguage; verbalization

Elise Vinel, Maitre de Conférences en Sciences de l'éducation à l'Université Paris 8, travaille sur les interactions et les pratiques langagières des enseignant.e.s et des élèves dans différents contextes scolaires.

Université Paris 8 Vincennes-Saint-Denis, Laboratoire Circeft-Escol, UFR SEPF, 2 rue de la Liberté, F-93526 Saint-Denis

E-Mail : Elise.vinel@univ-paris8.fr

Élisabeth Bautier est Professeure émérite en Sciences de l'éducation à l'université Paris 8, ses recherches portent sur les inégalités sociales d'apprentissages scolaires liées aux usages du langage.

Université Paris 8 Vincennes-Saint-Denis, Laboratoire Circeft-Escol, UFR SEPF, 2 rue de la Liberté, F-93526 Saint-Denis

E-Mail : bautier@wanadoo.fr 